\title{
Towards Animal-Free Neurotoxicity Screening: Applicability of hiPSC-Derived Neuronal Models for In Vitro Seizure Liability Assessment
}

\author{
Anke M. Tukker, Regina G. D. M. van Kleef, Fiona M. J. Wijnolts, Aart de Groot and Remco H. S. Westerink \\ Neurotoxicology Research Group, Toxicology Division, Institute for Risk Assessment Sciences (IRAS), Faculty of Veterinary Medicine, Utrecht University, \\ Utrecht, The Netherlands
}

\begin{abstract}
A sizeable proportion of drug attrition is due to drug-induced seizures. Currently available animal models frequently fail to predict human seizure liability. Therefore, there is a need for in vitro alternatives, preferably based on human-derived neurons, to circumvent interspecies translation. The increasing number of commercially available human induced pluripotent stem cell (hiPSC)-derived neuronal models holds great promise for replacing rodent primary cultures. We therefore tested three different hiPSC-derived neuronal models for their applicability for in vitro seizure liability assessment.

Using immunofluorescent staining and multi-well micro-electrode arrays, we show that all models develop functional neuronal networks that exhibit spontaneous activity and (network) bursting behavior. Development of activity patterns differed between the models, probably due to differences in model composition and seeding density. Neuronal activity and (network) bursting was reproducibly modulated with the seizurogenic compounds strychnine, picrotoxin (PTX) and 4-aminopyridine (4-AP). However, the sensitivity and degree of chemical-induced effects differed between the models, which can likely be explained by differences in seeding density, maturation and different ratios of inhibitory and excitatory cell types. Importantly, compared to rat primary cortical neurons, the hiPSC-derived neuronal models were equally, or even better in the case of 4-AP, suited to detect seizurogenicity. Overall, our data indicate that hiPSC-derived neuronal models may be used as a first screening tool for in vitro seizure liability assessment. However, before hiPSC-derived neuronal models can fully replace animal experiments, more compounds should be tested and the available models must be further characterized to fully understand their applicability.
\end{abstract}

\section{Introduction}

Drug development and safety testing are often done using in vivo and ex vivo experiments. These experiments are, however, not fully predictive of adverse effects in humans. While human drug approval rates have been increasing in recent years, only $10.4 \%$ of drugs in phase I of drug development will ultimately gain final approval (Hay et al., 2014). Most drugs fail the trajectory, often due to safety concerns related to the central nervous system (CNS). Even when compounds reach the market, drug attrition as a result of neurotoxicity and CNS problems remains a challenging concern (Onakpoya et al., 2016). An increased risk of seizures, i.e., periods of abnormal, rhythmic and uncontrolled hyper-excitability of neurons characterized by hyper-synchronicity of electrical activity (Easter et al., 2009; Jiruska et al., 2013), is amongst the most commonly encountered CNS-related problems during pre-clinical drug development (Authier et al., 2016). Drug-induced seizures can be life-threatening and are classified as severe adverse drug reactions. It is therefore of utmost importance that (increased) seizure liability can be detected early during drug development.

Since animal models frequently fail in human seizure liability predictions, their usefulness is debated (Little et al., 2019). Consequently, in vitro alternatives are required to reduce the number of animal tests and to improve safety screening. However, in vitro seizure liability testing is challenging because the CNS contains many different cell types with a diverse range of drug targets that can all have effects on brain function and excitability (Easter et al., 2009). In vitro models for seizure liability screening should thus ideally model the in vivo brain as closely as possible (Westerink, 2013).
Received July 12, 2019; Accepted October 28, 2019 Epub November 2, 2019; @ The Authors, 2019.

ALTEX 37(1), 121-135. doi:10.14573/altex.1907121

Correspondence: R.H.S. Westerink, PhD Neurotoxicology Research Group, Toxicology Division Institute for Risk Assessment Sciences (IRAS)

Faculty of Veterinary Medicine, Utrecht University

P.O. Box 80.177, 3508 TD Utrecht, The Netherlands

(r.westerink@uu.nl)
This is an Open Access article distributed under the terms of the Creative Commons Attribution 4.0 International license (http//creativecommons.org/licenses/by/4.0/) which permits unrestricted use, distribution and reproduction in any medium, provided the original work is appropriately cited. 
The most commonly used ex vivo model for seizure liability assessment in drug research is the brain slice assay (Authier et al., 2016). While the brain slice assay accurately mimics the in vivo organization of the brain (Grainger et al., 2018), including active and intact neuronal networks, the slices have a relatively short life-span (Buskila et al., 2015). More importantly, brain slices are of animal origin, and recordings require specific expertise and equipment, and are labor-intensive, thereby limiting high-throughput screening (Grainger et al., 2018).

The recent introduction of multiwell micro-electrode array (mwMEA) recordings eliminates some of these concerns. For this technique, cells are grown on a culture surface area with an integrated array of micro-electrodes, allowing non-invasive recordings of extracellular local field potentials simultaneously at different locations in the in vitro network (for review see Johnstone et al., 2010). MEA recordings yield a broad range of data on parameters that describe the state of the network. Parameters related to activity and synchronicity can be used to detect a hyper-active and/or hyper-synchronized state of the network that is linked to seizure-like events in the in vivo situation (Ishii et al., 2017; Bradley et al., 2018).

Many cell types can be grown on mwMEAs, but rat primary cortical cultures are the current gold standard (Authier et al., 2016). Primary cortical cultures grown on mwMEAs possess many characteristics of in vivo neurons, such as development of spontaneous network activity including (network) bursting (Cotterill et al., 2016) and responsiveness to neurotransmitters, pharmacological agents, and toxicological modulation (Hogberg et al., 2011; McConnell et al., 2012; Nicolas et al., 2014; Valdivia et al., 2014; Hondebrink et al., 2016). Rodent cortical cultures grown on mwMEAs have been used for seizure liability assessment with positive results as known seizurogenic compounds increased spiking, (network) bursting activity and/or synchronicity (Bradley et al., 2018; Kreir et al., 2018; Fan et al., 2019). Although the combination of mwMEA and rodent primary cultures is promising, it is still based on primary animal cells.

As also outlined in NC3Rs' Crack-It Neuratect Challenge ${ }^{1}$, there is a clear need for new in vitro drug screening models that are able to reliably detect seizures and can be used in a time and cost-efficient manner. These models should preferably be of human origin to circumvent interspecies translation and reduce the number of animal experiments. Human induced pluripotent stem cell (hiPSC)-derived neurons are not associated with ethical concerns like embryonic stem cells and animal experiments. It has been shown that hiPSC-derived neurons can be cultured on mwMEAs and exhibit spontaneous neuronal activity with (network) bursting behavior of mature neurons (Odawara et al., 2016; Paavilainen et al., 2018; Sasaki et al., 2019). These hiPSC-derived neurons can be modulated with known neurotoxicants and drugs (Tukker et al., 2016; Hondebrink et al., 2017; Odawara et al., 2018). However, one of the challenges in using hiPSC-derived neurons is the long time it takes to generate the neurons, which can vary from weeks (Kuijlaars et al., 2016) to months (Odawara et al., 2016), and the potential variability between batches of differentiated cells (Little et al., 2019). The introduction of commercially available hiPSC-derived neurons can help to overcome these concerns as they are quality-controlled, can be purchased in large quantities (Anson et al., 2011; Little et al., 2019), and can be used for neurotoxicity screening following a relatively short culture duration (Tukker et al., 2018).

With the increasing availability of hiPSC-derived neurons, there are also differences between the available models. Human neuronal models can differ, for example, in the ratio of excitatory and inhibitory neurons as well as in the presence or absence of astrocytes. These factors greatly affect the (bursting) behavior of the hiPSC-derived model (Tukker et al., 2018). Also, variations in the differentiation protocol as well as the level of maturation before freezing may affect the culture. This study aims to explore whether these models, despite their differences, are suitable for animal-free seizure liability testing. To that aim, we cultured three different commercially-available hiPSC-derived neuronal models to assess the development of spontaneous neuronal network activity. Subsequently, these models were challenged with known seizurogenic compounds and the results were compared to data from rat primary cortical cultures, which were exposed in parallel.

\section{Animals, materials and methods}

\subsection{Animals}

All experiments were conducted in accordance with Dutch law and were approved by the Ethical Committee for Animal Experiments of Utrecht University. Animals were treated humanely, and all efforts were made to alleviate suffering. Primary cultures of rat cortical neurons were prepared from postnatal day (PND) 0-1 pups of timed pregnant Wistar rat dams (Envigo, Horst, The Netherlands) as described in section 2.2.1.

\subsection{Chemicals}

Neurobasal ${ }^{\circledR}$-A medium, Dulbecco's Modified Eagle Medium (DMEM)-F12, fetal bovine serum (FBS), penicillin/streptomycin $(5000 \mathrm{U} / \mathrm{mL}(5000 \mu \mathrm{g} / \mathrm{mL})$ for rat primary cortical culture media and $10.000 \mathrm{U} / \mathrm{mL}(10.000 \mu \mathrm{g} / \mathrm{mL})$ for supplemented Brainphys $^{\mathrm{TM}}$ medium), B27 supplement, N2 supplement, L-glutamine, 4',6-diamidino-2-phenylindole (DAPI), donkey anti-rabbit Alexa Fluor ${ }^{\circledR} 488$ and donkey anti-mouse Alexa Fluor ${ }^{\circledR} 594$ were obtained from Life Technologies (Bleiswijk, The Netherlands). Goat anti-chicken Alexa Fluor ${ }^{\circledR} 647$ was obtained from Jackson ImmunoResearch Europe (Ely, UK). Paraformaldehyde (PFA) was obtained from Electron Microscopy Sciences (Hatfield, Pennsylvania, USA). Rabbit anti- $\beta$ (III)-tubulin (Ab18207), mouse anti-S100 $\beta$ (Ab11178) and chicken anti-MAP2 (Ab5392) were obtained from Abcam (Cambridge, United Kingdom). FluorSave was obtained from Calbiochem (San Diego, California, USA). iCell ${ }^{\circledR}$ Neural Supplement B and Nervous System Supplement were provided by Cellular Dynamics International (Madison, WI, USA). BrainPhys ${ }^{\mathrm{TM}}$ neuronal medium was ob-

1 https://www.NC3Rs.org.uk 
Tab. 1: Composition, density and exposure DIV of the different neuronal culture models

\begin{tabular}{|c|c|c|c|c|}
\hline Cell models & Cell types (\%) & $\begin{array}{l}\text { Excitatory: } \\
\text { inhibitory } \\
\text { neurons (ratio) }\end{array}$ & $\begin{array}{l}\text { Cells } \\
\text { seeded per } \\
\text { droplet }\end{array}$ & $\begin{array}{l}\text { Exposure } \\
\text { DIV }\end{array}$ \\
\hline $\begin{array}{l}\text { iCell } \|^{\circledR} \text { Glutaneurons - } \\
\text { iCeel } \|^{\circledR} \text { Astrocytes co-culture }\end{array}$ & $\begin{array}{l}\text { Neurons ( } 85 \% \text {, of which } 70 \% \text { glutamatergic and } \\
30 \% \text { GABAergic) / Astrocytes (15\%) }\end{array}$ & 2.3:1 & $140 \mathrm{k}$ & DIV14 \\
\hline CNS. $4 \mathrm{U}^{\circledR}$ co-culture & $\begin{array}{l}\text { Glutamatergic neurons }(\sim 40 \%) \text { / GABAergic } \\
\text { neurons }(\sim 40 \%) / \text { Dopaminergic neurons }(\sim 10 \%) \text { / } \\
\text { Astrocytes }(\sim 10 \%)^{\mathrm{a}}\end{array}$ & $1: 1$ & $36 k$ & DIV23 \\
\hline SynFire ${ }^{\circledR}$ iNs co-culture & $\begin{array}{l}\text { Glutamatergic neurons (52\%) / GABAergic } \\
\text { neurons }(22 \%) \text { / Astrocytes (26\%) }\end{array}$ & 2.3:1 & $270 k$ & DIV28 \\
\hline Rat primary cortical neurons & $\begin{array}{l}\text { Neurons }(55 \% \text {, of which } 70-80 \% \text { glutamatergic } \\
\left.\text { and } 20-30 \% \text { GABAergic }{ }^{\mathrm{b}}\right) / \text { Astrocytes }(45 \%)^{\mathrm{c}}\end{array}$ & $3: 1$ & $100 \mathrm{k}$ & DIV9-11 \\
\hline
\end{tabular}

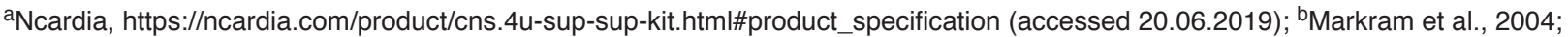

'Görtz et al., 2004; Tukker et al., 2016

tained from StemCell Technologies (Cologne, Germany). Neuro. $4 \mathrm{U}^{\circledR}$ basal medium $\mathrm{A}$ and CNS.4U ${ }^{\circledR}$ supplement were provided by Ncardia (Leiden, The Netherlands). SynFire ${ }^{\circledR}$ seeding basal medium, short-term basal medium, long-term basal medium and accessory supplements were provided by NeuCyte (Sunnyvale, CA, USA). Laminin (L2020), 50\% polyethyleneimine solution, sodium borate, boric acid and all other chemicals (unless stated otherwise) were obtained from Sigma-Aldrich (Zwijndrecht, The Netherlands).

Stock solutions of strychnine $\mathrm{HCl}$ were made in purity-checked dimethyl sulfoxide (DMSO) and stored at $4{ }^{\circ} \mathrm{C}$. 4-Aminopyridine (4-AP) was freshly dissolved in medium on the day of the experiment. Stock solutions of picrotoxin (PTX) were made in ethanol (EtOH; VWR, Amsterdam, The Netherlands) on the day of the experiment. Final concentration of the solvent in exposure solutions was kept at or below $0.1 \%$ ( $\mathrm{vol} / \mathrm{vol})$.

\subsection{Cell culture}

Primary rat cortical cultures and all hiPSC-derived neuronal co-cultures were kept at $37^{\circ} \mathrm{C}$ in a humidified $5 \% \mathrm{CO}_{2}$ incubator. 48-well MEA plates (Axion BioSystems Inc., Atlanta, GA, USA) and $\mu$-slide 8 -well chambered coverslips (Ibidi GmbH, Planegg, Germany) were pre-coated with $0.1 \%$ polyethyleneimine solution diluted in borate buffer $(24 \mathrm{mM}$ sodium borate, $50 \mathrm{mM}$ boric acid in Milli-Q, pH adjusted to 8.4). See Table 1 for an overview of the culture details of the four different models, including the day on which their chemical sensitivity was assessed (exposure DIV). Notably, the exposure days used to determine the drug response differed between hiPSC-derived neuronal models as a consequence of the manufacturers' recommendations.

iCell ${ }^{\circledR}$ Glutaneurons - iCell ${ }^{\circledR}$ Astrocytes co-culture

iCell $^{\circledR}$ Glutaneurons (Lot\# 103288; Cellular Dynamics International, Madison, WI, USA) and iCell ${ }^{\circledR}$ Astrocytes (Lot\# 11493, 1414 and 1444; Cellular Dynamics International, Madison, WI, USA) were thawed and cultured according to the manufacturer's protocol. Briefly, each cell type was thawed separately in sup- plemented BrainPhys ${ }^{\mathrm{TM}}$ medium (BrainPhys ${ }^{\mathrm{TM}}$ medium supplemented with 2\% iCell ${ }^{\circledR}$ Neural Supplement B, 1\% Nervous System Supplement, $1 \%$ N2, $1 \%$ penicillin/streptomycin and $0.1 \%$ laminin). The cell pellet was diluted in dotting medium (supplemented BrainPhys ${ }^{\mathrm{TM}}$ medium with $10 \%$ laminin) at a density of $15 \mathrm{k}$ cells $/ \mu \mathrm{L}$ for iCell ${ }^{\circledR}$ Glutaneurons or $6.6 \mathrm{k}$ cells $/ \mu \mathrm{L}$ for $\mathrm{iCell}^{\circledR}$ Astrocytes. Before plating, iCell ${ }^{\circledR}$ Glutaneurons and iCell ${ }^{\circledR}$ Astrocytes were premixed to a co-culture containing $120 \mathrm{k}$ iCell ${ }^{\circledR} \mathrm{Glu}$ taneurons $(85 \%)$ and $20 \mathrm{k} \mathrm{iCell}{ }^{\mathbb{R}}$ Astrocytes $(15 \%)$ and then plated in $11 \mu \mathrm{L}$ droplets (140k cells/droplet) over the electrode field of pre-coated MEA wells or in a chamber of the $\mu$-slide coverslip. Cells were allowed to adhere for $\sim 1 \mathrm{~h}$ before $300 \mu \mathrm{L}$ (MEA) or $200 \mu \mathrm{L}$ (coverslip) room temperature (RT) supplemented BrainPhys $^{\mathrm{TM}}$ medium was added. 50\% Medium changes with RT supplemented BrainPhys ${ }^{\mathrm{TM}}$ medium took place at DIV1, 2, 4, 6, 8, 10,12 and 14.

CNS.4U ${ }^{\circledR}$ culture

CNS.4U ${ }^{\circledR}$ (Lot\# CN39CL_V_2M and CN59CL_V-x2; Ncardia, Leiden, The Netherlands) were obtained as a mixture of $\sim 40 \%$ glutamatergic neurons, $\sim 40 \%$ GABAergic neurons, $\sim 10 \%$ dopaminergic neurons and $\sim 10 \%$ astrocytes. Cells were thawed and cultured according to the manufacturer's protocol. Briefly, a vial was thawed in Neuro. $4 \mathrm{U}^{\circledR}$ basal medium A, and the cell pellet was dissolved in complete Neuro. $4 \mathrm{U}^{\circledR}$ medium A (basal medium and supplement) at a density of $12 \mathrm{k}$ cells $/ \mu \mathrm{L}$. Cells were plated in $3 \mu \mathrm{L}$ droplets ( $36 \mathrm{k}$ cells in total) directly over the electrode field of pre-coated MEA wells or in the chamber of a $\mu$-slide coverslip. Cells were allowed to adhere for $\sim 1 \mathrm{~h}$ before $300 \mu \mathrm{L}$ (MEA) or $200 \mu \mathrm{L}$ (coverslip) RT Neuro.4U ${ }^{\circledR}$ medium was added. At DIV1, 7, 14 and 21, 100\% medium changes were performed with complete Neuro.4U ${ }^{\circledR}$ medium, and at DIV2, 4, 9, 11, 16 and 18, 50\% of the medium was replaced with complete Neuro. $4 \mathrm{U}^{\circledR}$ medium.

SynFire ${ }^{\circledR}$ iNs co-culture

SynFire $^{\circledR}$ glutamatergic neurons (Lot\#104, 109 and 116), SynFire $^{\circledR}$ GABAergic neurons (Lot\#105, 109, 110 and 116) and 
SynFire $^{\circledR}$ astrocytes (Lot\#12854 and 13029; all from NeuCyte, Sunnyvalle, CA, USA) were thawed and cultured according to the manufacturer's protocol. In short, each cell type was thawed separately in DMEM-F12 medium. The cell pellet was dissolved in complete seeding medium (containing seeding supplement) at a density of $10 \mathrm{k}$ cells $/ \mu \mathrm{L}$ (for all cell types). Next, a mixture was made containing $140 \mathrm{k}$ glutamatergic neurons $(52 \%$ of total cell number), 60k GABAergic neurons (22\%) and 70k astrocytes $(26 \%)$. The mixture was plated in $50 \mu \mathrm{L}$ droplets (270k cells in total) over the electrode field of pre-coated MEA wells or in a chamber of the $\mu$-slide coverslip. Cells were left overnight to adhere. On the next day (DIV1), $250 \mu \mathrm{L}$ (MEA) or $150 \mu \mathrm{L}$ (coverslip) RT complete short-term maintenance medium (containing short-term supplement) was added. At DIV3 and 5, 50\% medium changes with complete short-term maintenance medium took place. The remaining 50\% medium changes at DIV7, 10, 13, 16, 19,22 and 25 were performed with RT complete long-term maintenance medium (containing long-term supplement A and B).

\section{Rat primary cortical culture}

Primary rat cortical cells were isolated from PND0-1 Wistar rat pups as described previously (Dingemans et al., 2016; Tukker et al., 2016). Briefly, PND0-1 pups were decapitated and cortices were rapidly dissected on ice and kept in dissection medium (Neurobasal ${ }^{\circledR}$-A supplemented with $25 \mathrm{~g} / \mathrm{L}$ sucrose, $450 \mu \mathrm{M} \mathrm{L}$ glutamine, $30 \mu \mathrm{M}$ glutamate, $1 \%$ penicillin/streptomycin and $10 \%$ FBS, pH 7.4) during the entire procedure. Cortices were dissociated to a single-cell suspension by mincing with scissors, trituration and filtering through a $100 \mu \mathrm{m}$ mesh (EASYstrainer, Greiner). The cell suspension was diluted to $2 \times 10^{6}$ cells $/ \mathrm{mL}$. Droplets of $50 \mu \mathrm{L}$ (100k cells in total) were placed on the electrode fields in wells of pre-coated MEA plates. Cells were left to adhere for $\sim 2 \mathrm{~h}$ before adding $450 \mu \mathrm{L}$ dissection medium. At DIV2, $90 \%$ of the dissection medium was replaced with glutamate medium (Neurobasal ${ }^{\mathbb{R}}$-A supplemented with $25 \mathrm{~g} / \mathrm{L}$ sucrose, $450 \mu \mathrm{M} \mathrm{L}$-glutamine, $30 \mu \mathrm{M}$ glutamate, $1 \%$ penicillin/streptomycin and $2 \%$ B27 supplement, $\mathrm{pH}$ 7.4) to prevent glial overgrowth. At DIV4, $90 \%$ of the glutamate medium was replaced with FBS medium (Neurobasal ${ }^{\circledR}$-A supplemented with $25 \mathrm{~g} / \mathrm{L}$ sucrose, $450 \mu \mathrm{M}$ L-glutamine, $1 \%$ penicillin/streptomycin and $10 \% \mathrm{FBS}, \mathrm{pH} 7.4$ ).

\subsection{Immunocytochemistry}

The $\mu$-slide coverslips with different hiPSC-derived neuronal co-cultures were fixated on the day of exposure with $4 \%$ PFA in $0.1 \mathrm{M}$ PBS ( $\mathrm{pH}$ 7.4) for $15 \mathrm{~min}$ at RT. Following fixation, coverslips were quenched for PFA, permeabilized and incubated for $20 \mathrm{~min}$ at RT with $20 \mathrm{mM} \mathrm{NH}_{4} \mathrm{Cl}$ in blocking buffer $(2 \%$ bovine serum albumin and $0.1 \%$ saponin in PBS) as described previously (Tukker et al., 2019). Hereafter, coverslips were incubated overnight at $4^{\circ} \mathrm{C}$ with rabbit anti- $\beta \mathrm{III}$ tubulin (final dilution 1:500), mouse anti-S100ß (final dilution 1:500) and chicken anti-MAP2 (final dilution 1:1000) in blocking buffer. Next, coverslips were washed thrice with blocking buffer and incubated with donkey anti-rabbit Alexa Fluor ${ }^{\circledR} 488$, donkey anti-mouse Alexa Fluor ${ }^{\circledR} 594$ and goat anti-chicken Alexa Fluor ${ }^{\circledR} 647$ (final dilution 1:100) for $45 \mathrm{~min}$ at RT in the dark. During the last 2-3 min of this incubation,
$200 \mathrm{nM}$ DAPI for nuclear staining was added. Coverslips were again washed thrice with blocking buffer and sealed with 1-2 drops of FluorSave. The coverslips were stored in the dark at $4{ }^{\circ} \mathrm{C}$ until further use. Immunostained coverslips were visualized with a Leica SPEII Confocal microscope (Leica DMI4000 equipped with TCS-SPE-II) using a 40x oil immersion objective (ACS APO IMM NA 0.6). Images were captured as .lif files using Leica Application Suite Advanced Fluorescence software (LAS AF version 2.6.0; Leica Microsystems GmbH, Wetzlar, Germany).

\subsection{MEA measurements}

Each well of a 48-well MEA plate contains 16 nanotextured gold micro-electrodes $(\sim 40-50 \mu \mathrm{m}$ diameter; $350 \mu \mathrm{m}$ spacing $)$ with 4 integrated ground electrodes, yielding a total of 768 channels that can be recorded simultaneously. Spontaneous electrical activity was recorded on the days of medium changes and on the day of exposure (Tab. 1) as described previously (Nicolas et al., 2014; Tukker et al., 2019). In short, signals were recorded using a Maestro 768-channel amplifier with integrated heating system and temperature controller and a data acquisition interface (Axion BioSystems Inc., Atlanta, GA, USA). Data acquisition was managed with Axion's Integrated Studio (AxIS 2.4.2.13) and recorded as .RAW files. All channels were sampled at the same time with a gain of 1200x and a sampling frequency of $12.5 \mathrm{kHz} /$ channel with a $200-5000 \mathrm{~Hz}$ band-pass filter. Prior to the recording, MEA plates were allowed to equilibrate for $\sim 10 \mathrm{~min}$ in the Maestro.

To determine effects of the known seizurogenic compounds PTX, 4-AP and strychnine on spontaneous activity (spiking and (network) bursting behavior) of the different cell cultures, activity was recorded prior to exposure to generate a baseline recording. Immediately following this recording, cells were exposed to the seizurogenic compounds or the appropriate solvent control, and activity was recorded for another $30 \mathrm{~min}$ plus the time it took to expose all wells. Concentrations tested were determined by the NC3Rs' CRACK-it team based on the list from the HESI NeuTox MEA Subteam, and were in line with earlier studies assessing seizure liability (Kreir et al., 2018). Each well was exposed to only one single concentration of one compound in order to prevent receptor (de)sensitization or other compensatory cellular processes. For each experimental condition, MEA plates from at least two different plating rounds or culture preparations were used.

\subsection{Data analysis and statistics}

To determine (modulation of) spontaneous activity, .RAW data files were re-recorded to obtain Alpha Map files. In this re-recording, spikes were detected with the AxIS spike detector (Adaptive threshold crossing, Ada BandFIt v2) and a variable threshold spike detector set at $7 \mathrm{x}$ (rat primary cortical cultures) or $5.5 \mathrm{x}$ (all hiPSC-derived neuronal cultures) standard deviation (SD) of internal noise level (rms) on each electrode. Post/pre-spike duration was set to $3.6 / 2.4 \mathrm{~ms}$, respectively. For further data analysis, spike files were loaded in NeuralMetric Tool (version 2.2.4, Axion BioSystems), and only active electrodes (MSR $\geq 0.1$ spikes/s) in active wells ( $\geq 1$ active electrode) were included in the data analysis. Bursts were extracted with the Poisson Surprise method (Legéndy and Salcman, 1985) with a minimal surprise of 
Tab. 2: Overview and description of different metric parameters Adapted from Tukker et al. (2018).

\begin{tabular}{|c|c|}
\hline Metric parameter & Description \\
\hline Mean spike rate (MSR) & Total number of spikes divided by recording time $(\mathrm{Hz})$ \\
\hline ISI coefficient of variation & $\begin{array}{l}\text { Standard deviation ISI (inter-spike interval) divided by the mean ISI. Measure for spike } \\
\text { regularity: } 0 \text { indicates perfect spike distribution, }>1 \text { signals bursting }\end{array}$ \\
\hline Number of active electrodes & Average number of electrodes with a spike rate of at least 0.1 spike/s \\
\hline Number of active wells & Number of wells that exhibits activity that meets spike criteria \\
\hline Mean burst rate (MBR) & Total number of bursts divided by recording time $(\mathrm{Hz})$ \\
\hline Number of bursting electrodes & $\begin{array}{l}\text { Average number of electrodes in the well with number of bursts/second that is higher } \\
\text { than the burst criterion of } 0.005 \text { bursts/s }\end{array}$ \\
\hline Number of bursting wells & Number of wells that meets the bursting criteria \\
\hline Burst duration & Average time from the first spike in a burst till the last spike (s) \\
\hline Number of spikes per burst & Average number of spikes occurring in a burst \\
\hline Mean inter-spike interval (ISI) within burst & Mean inter-spike interval within a burst (s) \\
\hline Inter-burst interval (IBI) & Time between the last spike of a burst and the first spike of a subsequent burst (s) \\
\hline IBI coefficient of variation & Standard deviation of IBI divided by the mean IBI. Measure for burst regularity \\
\hline Burst percentage & Percentage of total number of spikes occurring in a burst \\
\hline Mean network burst rate (MNBR) & Total number of network bursts divided by recording time $(\mathrm{Hz})$ \\
\hline Network burst duration & Average time from the first spike till the last spike in a network burst (s) \\
\hline Number of spikes per network burst & Average number of spikes occurring in a network burst \\
\hline Mean ISI within network burst & Average of the mean ISIs within a network burst (s) \\
\hline $\begin{array}{l}\text { Number of electrodes participating in } \\
\text { network burst }\end{array}$ & Average number of electrodes with spikes that participate in the network burst \\
\hline Number of network bursting wells & Number of wells that meets the network burst criteria \\
\hline Network burst percentage & Percentage of total spikes occurring in a network burst \\
\hline Network IBI coefficient of variation & $\begin{array}{l}\text { Standard deviation of network IBI divided by the mean network IBI. Measure of network } \\
\text { burst rhythmicity: value is small when bursts occur at regular interval and increases when } \\
\text { bursts occur more sporadically }\end{array}$ \\
\hline Network normalized duration IQR & $\begin{array}{l}\text { Interquartile range of network burst durations. Measure for network burst duration } \\
\text { regularity: larger values indicate wide variation in duration. }\end{array}$ \\
\hline Area under normalized cross-correlation & $\begin{array}{l}\text { Area under inter-electrode cross-correlation normalized to the auto-correlations. } \\
\text { The higher the value, the greater the synchronicity of the network. }\end{array}$ \\
\hline $\begin{array}{l}\text { Full width at half height }(\mathrm{FWHH}) \text { of } \\
\text { normalized cross-correlation }\end{array}$ & $\begin{array}{l}\text { Width at half height of the normalized cross-correlogram. Measure for network synchrony: } \\
\text { the higher the value, the less synchronized the network is. }\end{array}$ \\
\hline
\end{tabular}

10 and a minimum bursting frequency of 0.005 bursts/s. Network bursts were determined with an adaptive threshold algorithm.

For developmental curves, full recordings from the days of medium change and the baseline recording of the day of exposure were used for data analysis, and the means of different network parameters (Tab. 2) were calculated based on well averages. The percentage of active wells depicts the percentage of wells that fulfil the activity criteria relative to the total number of seeded wells. The percentage of bursting wells depicts the percentage of wells that meet the bursting criteria and is calculated with respect to the number of active wells. Finally, the percentage of network bursting wells is the percentage of wells that exhibit network bursts relative to the number of bursting wells. This implicates that only the most active wells are included in the network burst developmental analysis, automatically resulting in a relatively high number of network bursting electrodes.

The effects of seizurogenic compounds on spontaneous activity were determined by comparing the baseline activity with activity following exposure. A custom-made MS Excel macro was used to calculate treatment ratios (TR) per well for the different met- 

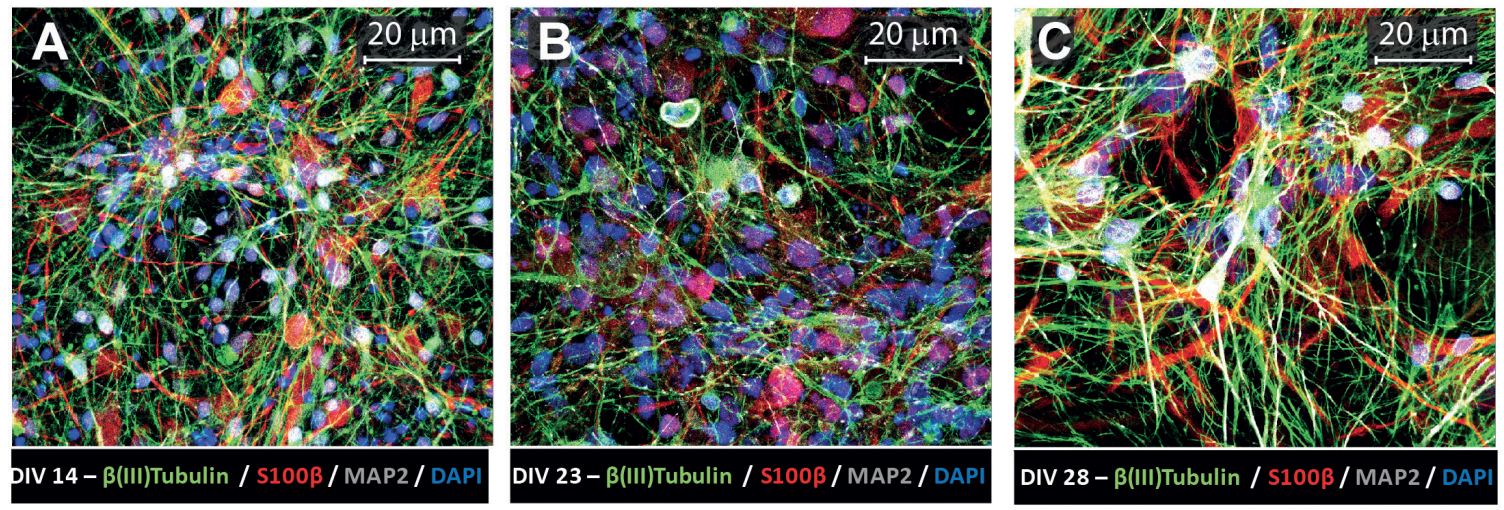

Fig. 1: Immunofluorescent images of iCell ${ }^{\circledR}$ Glutaneurons - iCell ${ }^{\circledR}$ Astrocytes co-culture (A), CNS.4U ${ }^{\circledR}$ co-culture (B) and SynFire ${ }^{\circledR}$ iNs co-culture (C) illustrating (neuronal) network structures with MAP2 (mature neurons; grey), $\beta$ (III) tubulin (general neuronal marker; green) and $\mathrm{S} 100 \beta$ (astrocytes; red)

Nuclei are stained with DAPI (blue). Scale bar depicts $20 \mu \mathrm{m}$.

ric parameters (Tab. 2) by: (parameter ${ }_{\text {exposure }} /$ parameter $_{\text {baseline }}$ ) x $100 \%$. Hereafter, TRs were normalized to appropriate vehicle control (medium, DMSO or EtOH). To prevent inclusion of exposure artefacts, effect analysis was performed in the window of 20-30 min post-exposure for all tested compounds.

Wells that showed an effect $\geq 2 x$ SD above or below average were considered outliers and removed from further data analysis $\left(4 \%\right.$ for iCell ${ }^{\circledR}$ Glutaneurons - iCell ${ }^{\circledR}$ Astrocytes co-culture; $3 \%$ for CNS. $4 \mathrm{U}^{\circledR}$ co-culture; $4 \%$ for SynFire ${ }^{\circledR}$ iNs co-culture and $5 \%$ for rat primary cortical culture). Concentration-dependent effects were determined by one-way Welch ANOVA followed by a post hoc Dunnett test. $P$-values $<0.05$ were considered statistically significant. All statistical analyses were performed in $\mathrm{R}$ version 3.6.0 $0^{2}$ using the DescTools package version 0.99.28 3 . Spider plots and heat maps were created in $\mathrm{R}$ with the packages fmsb version $0.6 .3^{4}$ and pheatmap version $1.0 .12^{5}$, respectively. All data are presented as mean \pm standard error of the mean (SEM) from the number of wells (n) indicated, derived from at least 2 independent plating rounds $(\mathrm{N})$.

\section{Results}

\subsection{Immunofluorescent staining of the different hiPSC-derived neuronal models}

As a first characterization, the three different hiPSC-derived neuronal co-cultures were labelled with microtubule-associated protein 2 (MAP2, marker for mature neurons), $\beta$ (III)tubulin (general neuronal marker) and S100 calcium binding protein $\beta$ (S100 $\beta$, marker for astrocytes) antibodies to visualize the heterogeneity and complexity of the in vitro networks (Fig. 1). The iCell ${ }^{\circledR}$ Glutaneurons - iCell ${ }^{\circledR}$ Astrocytes co-culture (A), CNS.4U ${ }^{\circledR}$ co-culture (B) and SynFire ${ }^{\circledR}$ iNs co-culture (C) all showed network formation with a high degree of complexity at their corresponding days of exposure (Tab. 1). Images also indicate that neurons and astrocytes were spread evenly through the cultures.

These results show that all three different hiPSC-derived neuronal co-cultures consisted of mature neurons and astrocytes and indicate that neuronal networks were formed. The network complexity and heterogeneity were comparable to the degree of complexity present in rat primary cortical culture (Hondebrink et al., 2016).

\subsection{Development of spontaneous neuronal activity and (network) bursting behavior}

The different hiPSC-derived neuronal models were cultured according to continuously improving manufacturer's recommendations up to DIV14 (iCell ${ }^{\circledR}$ Glutaneurons - iCell ${ }^{\circledR}$ Astrocytes co-culture), DIV23 (CNS.4U ${ }^{\circledR}$ co-culture) or DIV 28 (SynFire $^{\circledR}$ iNs co-culture) on mwMEAs to assess the development of spontaneous neuronal network activity and bursting behavior over time. All models developed spontaneous activity (Fig. 2).

The mean spike rate (MSR) was relatively stable for the $\mathrm{iCell}^{\circledR}$ Glutaneurons - $\mathrm{iCell}^{\circledR}$ Astrocytes co-culture and CNS.4U ${ }^{\circledR}$ co-culture (Fig. 2A,B, left), whereas the MSR of the SynFire ${ }^{\circledR}$ iNs co-culture increased over the entire culture period (Fig. $2 \mathrm{C}$, left). The percentage of spiking wells is stable and comparable $(>75 \%)$ for all models.

\footnotetext{
2 R: A language and environment for statistical computing. R Foundation for Statistical Computing, Vienna, Austria. https://www.R-project.org/

3 DescTools: Tools for Descriptive Statistics. https://cran.r-project.org/package=DescTools

$4 \mathrm{fmsb}$ : Functions for Medical Statistics Book with some Demographic Data. https://CRAN.R-project.org/package=fmsb

5 Pheatmap: Pretty Heatmaps. https://CRAN.R-project.org/package=pheatmap
} 


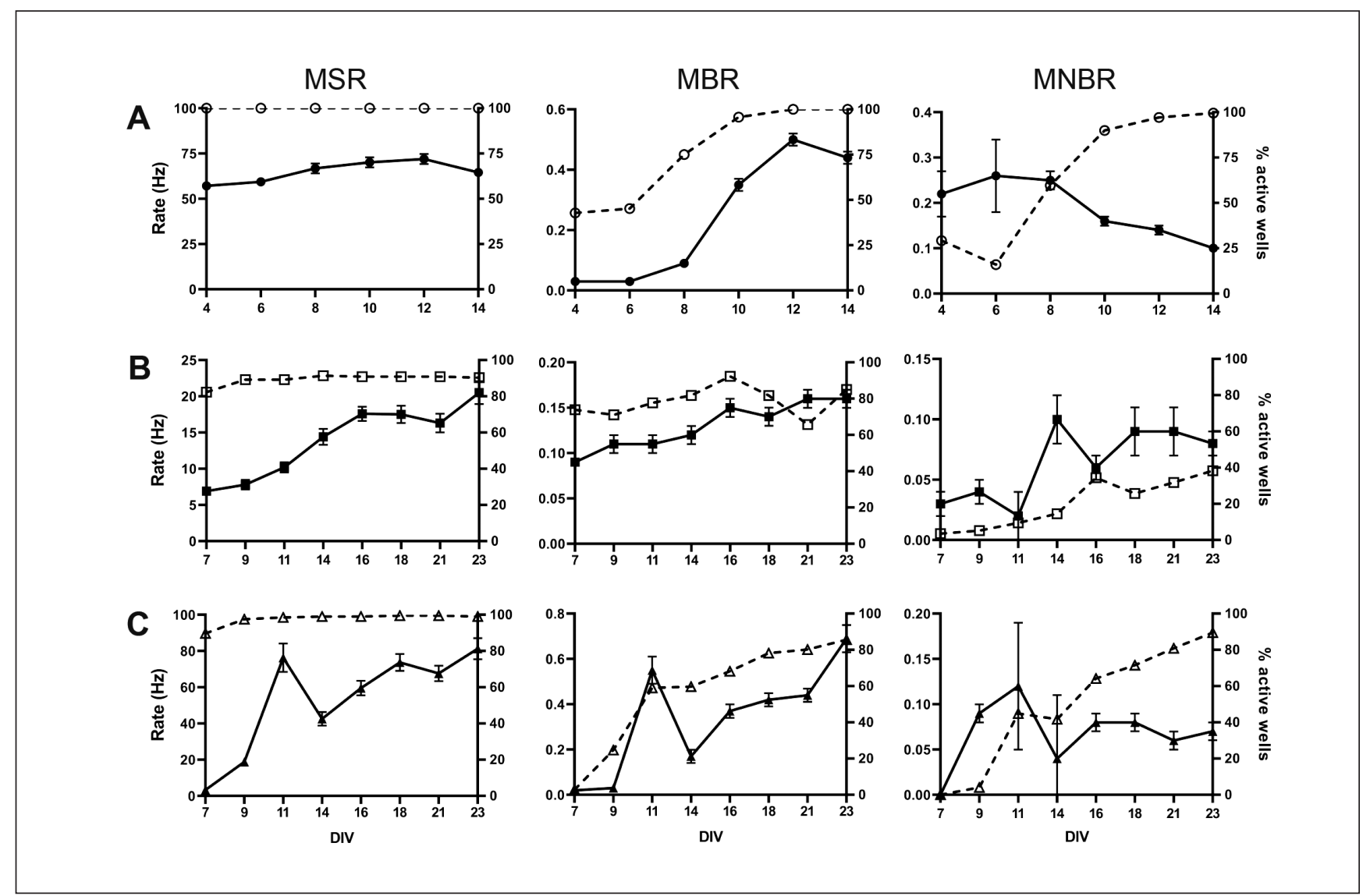

Fig. 2: Development of mean spike rate (MSR; left), mean burst rate (MBR; middle), and mean network burst rate (MNBR: right) in solid lines and percentage of spiking wells (left), bursting wells (middle), and network bursting wells (right) in dashed lines for iCell ${ }^{\circledR}$ Glutaneurons - iCell ${ }^{\circledR}$ Astrocytes co-culture (A), CNS.4U ${ }^{\circledR}$ co-culture (B) and SynFire ${ }^{\circledR}$ iNs co-culture (C)

Data are expressed as mean \pm SEM from $n=26-208$ wells, $N=5$ plates for the iCell ${ }^{\circledR}$ Glutaneurons - iCell ${ }^{\circledR}$ Astrocytes co-culture; $\mathrm{n}=4-170$ wells, $\mathrm{N}=5$ plates for the CNS. $4 \mathrm{U}^{\circledR}$ co-culture and $\mathrm{n}=2-202$ wells, $\mathrm{N}=8$ plates for the SynFire ${ }^{\circledR}$ iNs co-culture.

The mean burst rate (MBR) of the CNS.4U ${ }^{\circledR}$ co-culture was stable during the whole culture period, however the rate was low compared to that of the other two models, especially at the day of exposure (Fig. 2B, middle). The SynFire ${ }^{\circledR}$ iNs co-culture had the highest MBR at the end of the culture period (Fig. 2C, middle). The percentage of bursting wells was $>75 \%$ for all three models at the day of exposure.

At the beginning of the culture period, the iCell ${ }^{\circledR}$ Glutaneurons - iCell ${ }^{\circledR}$ Astrocytes co-culture had a high mean network burst rate (MNBR; Fig. 2A, right), especially compared to the other two models (Fig. 2B, C, right). However, this rate decreased rapidly over time to levels comparable with the other 2 models. At the day of exposure, all cultures exhibited a comparable MNBR, but the percentage of wells that exhibited network bursts was low in the CNS. $4 \mathrm{U}^{\circledR}$ co-culture $(<50 \%)$ compared to the other two models $(>75 \%)$.

Further analysis of additional (network) burst related parameters revealed additional differences between the models, as is depicted in spider plots (Fig. 3). It becomes clear from the layout of these graphs that the models developed in a different man- ner. Whereas the burst duration became shorter during prolonged culture of the iCell ${ }^{\circledR}$ Glutaneurons - iCell ${ }^{\circledR}$ Astrocytes co-culture (Fig. 3A), it increased slightly for the other two models (Fig. $3 \mathrm{~B}, \mathrm{C})$. For the iCell ${ }^{\circledR}$ Glutaneurons $-\mathrm{iCell}^{\circledR}$ Astrocytes co-culture (Fig. 3A) and the CNS.4U ${ }^{\circledR}$ co-culture (Fig. 3B), the number of spikes per network burst and network burst percentage increased over time, in contrast to the SynFire ${ }^{\circledR}$ iNs co-culture (Fig. 3C) where this pattern was relatively stable. The interburst interval (IBI) was longest in early culture DIVs for the iCell ${ }^{\circledR}$ Glutaneurons - iCell ${ }^{\circledR}$ Astrocytes co-culture and the SynFire ${ }^{\circledR}$ iNs co-culture, whereas for the CNS. $4 \mathrm{U}^{\circledR}$ co-culture, the IBI was relatively stable during the entire culture period. The number of spikes per burst increased during the culture period, resulting in an increasing burst percentage for the iCell ${ }^{\circledR}$ Glutaneurons iCell ${ }^{\circledR}$ Astrocytes co-culture and the SynFire ${ }^{\circledR}$ iNs, whereas this was relatively stable for the $\mathrm{CNS} .4 \mathrm{U}^{\circledR}$ co-culture.

The pattern of spontaneous electrical activity at the exposure DIV, the day in vitro at which the chemical sensitivity of the compound was assessed, is depicted in spike raster plots (Fig. 4) and further described by different metric parameters (Tab. 3). 


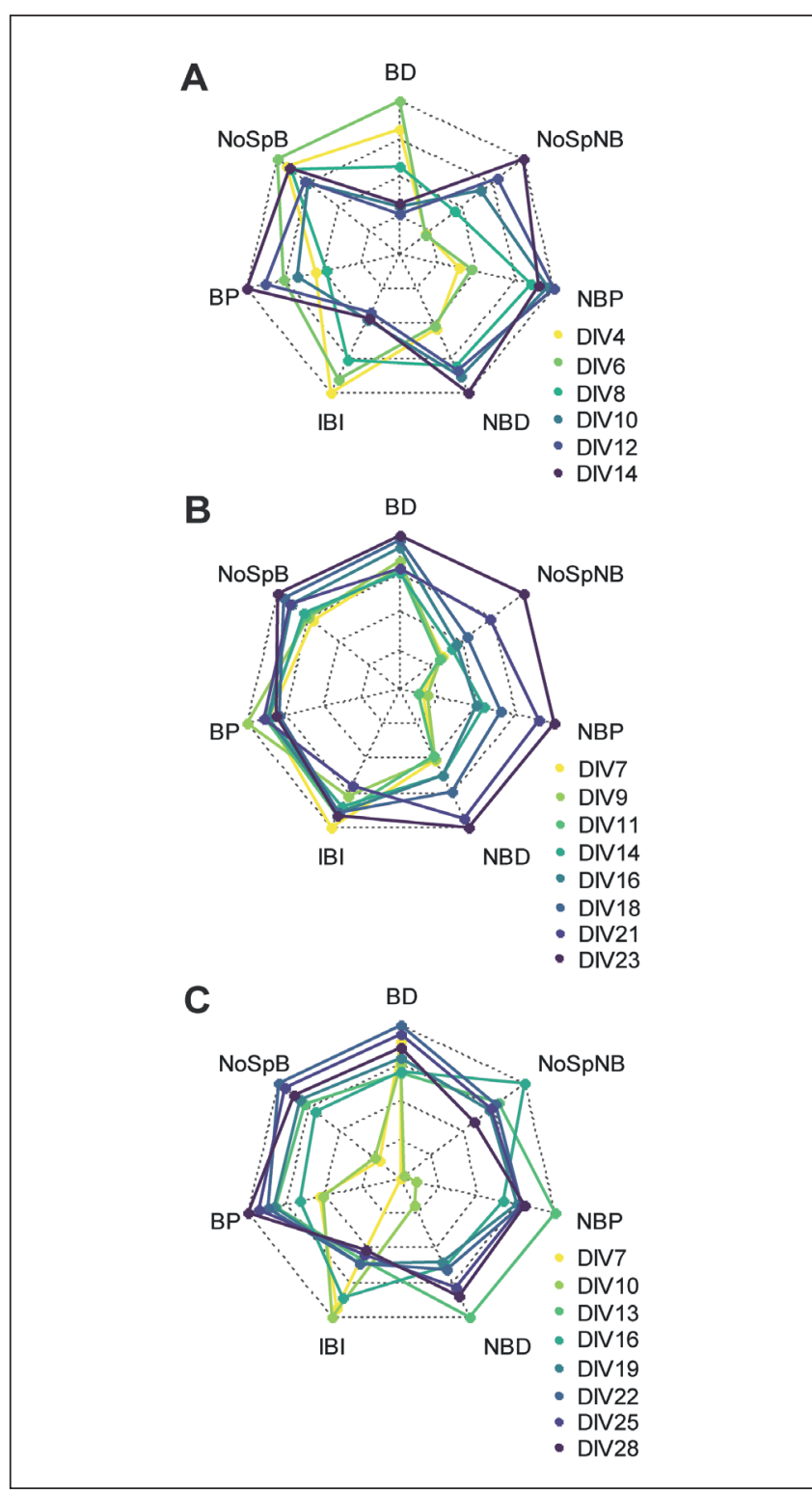

Fig. 3: Spider plots illustrating the differences in development of neuronal activity between the iCell ${ }^{\circledR}$ Glutaneurons - iCell ${ }^{\circledR}$ Astrocytes co-culture (A), CNS.4U ${ }^{\circledR}$ co-culture (B) and SynFire ${ }^{\circledR}$ iNs co-culture (C)

The yellow and lighter green lines depict earlier developmental DIVs, whereas darker shades belong to DIVs later in development. The grey grid represents $0 \%$ at the center, whereas the outer ring represents $100 \%$. For each model, the highest value of a parameter during the culture period of that model is set to $100 \%$. The axes represent (counter clockwise): burst duration (BD), number of spikes per burst (NoSpB), burst percentage (BP), inter-burst interval (IBI), network burst duration (NBD), network burst percentage (NBP) and number of spikes per network burst (NoSpNB), from $n=15-208$ wells, $N=5$ plates for the iCell ${ }^{\circledR}$ Glutaneurons - iCell ${ }^{\circledR}$ Astrocytes co-culture; $n=4-167$ wells, $N=5$ plates for the $\mathrm{CNS} .4 \mathrm{U}^{\circledR}$ co-culture; $\mathrm{n}=2-202$ wells, $\mathrm{N}=8$ plates for the SynFire ${ }^{\circledR}$ iNs co-culture.

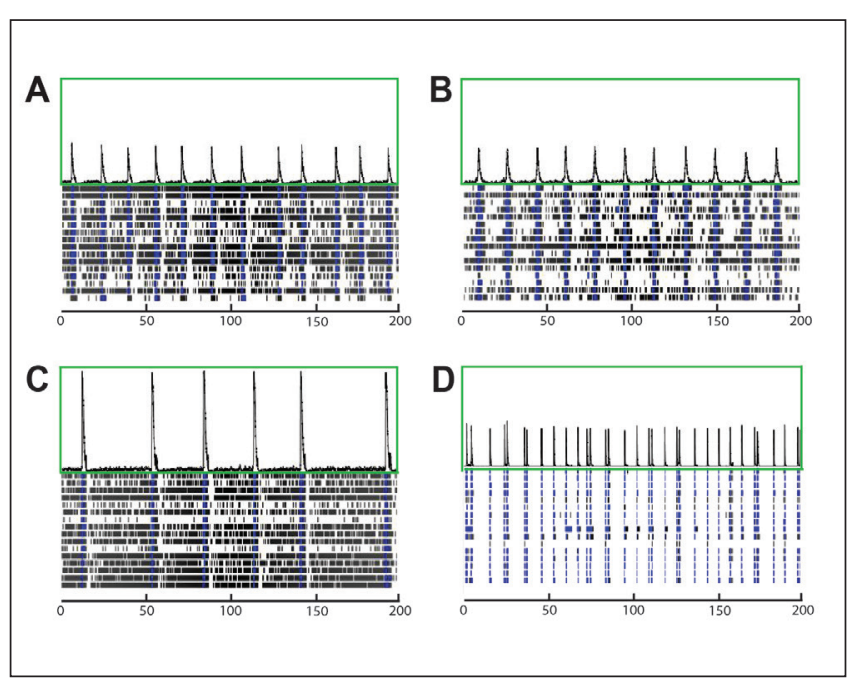

Fig. 4: Spike raster plots of the different hiPSC-derived co-cultures

Each row depicts one electrode, each tick mark represents one spike in a $200 \mathrm{~s}$ interval illustrating the pattern of activity of the iCell ${ }^{\circledR}$ Glutaneurons - iCell ${ }^{\circledR}$ Astrocytes co-culture $(A)$, the CNS.4U ${ }^{\circledR}$ co-culture (B), the SynFire ${ }^{\circledR}$ iNs co-culture (C), and the primary rat cortical neurons (D) at the exposure DIV. The cumulative trace (green box) above each plot depicts the population spike time histogram, indicating synchronized activity between the different electrodes.

The spike raster plots show that all three models exhibited a mature phenotype with synchronized firing with (network) bursts. The raster plots also confirm that the pattern and level of activity differed between the models. The higher MSR for the iCell ${ }^{\circledR}$ Glutaneurons - iCell ${ }^{\circledR}$ Astrocytes co-culture (Fig. 4A) and SynFire ${ }^{\circledR}$ iNs co-culture (Fig. 4C), as described in Table 3 and Figure 2, is also visible in the raster plots. The spike time histogram (green box, Fig. 4) clearly shows the different levels of synchronicity that are outlined in Table 3 , and indicate the high number of spikes within a network burst in the SynFire ${ }^{\circledR}$ iNs co-culture. Further details regarding the other developmental days of the hiPSC-derived co-cultures can be found in the supplementary material $^{6}$ (Tab. S1-S3 and Fig. S1).

As can be seen in the raster plots, rat primary cortical neurons exhibited a spike frequency comparable to the CNS.4U ${ }^{\circledR}$ co-culture (Fig. 4D, Tab. 3), whereas the burst frequency was more in line with the SynFire ${ }^{\circledR}$ iNs co-culture. Network burst activity of rat primary cortical neurons occurred in a frequency comparable to the CNS. $4 \mathrm{U}^{\circledR}$ co-culture and the SynFire ${ }^{\circledR}$ iNs co-culture. The burst duration of the primary rodent culture was much lower than in the hiPSC-derived co-cultures. In rat primary cortical neurons, most spikes were incorporated in (network) bursts as is reflected in the (network) burst percentage. This is in sharp contrast with hiPSC-derived neuronal models, where single spikes occurred.

6 doi:10.14573/altex.1907121s 
Tab. 3: Metric parameters at the exposure DIV (mean \pm SEM)

\begin{tabular}{|c|c|c|c|c|}
\hline Metric parameter (unit) & $\begin{array}{l}\text { iCell® } \\
\text { Glutaneurons - } \\
\text { iCell@ Astrocytes } \\
\text { (DIV14) }\end{array}$ & $\begin{array}{l}\text { CNS.4Uß } \\
\text { (DIV23) }\end{array}$ & $\begin{array}{l}\text { SynFire }{ }^{\circledR} \text { iNs } \\
\text { (DIV28) }\end{array}$ & $\begin{array}{l}\text { Rat cortical neurons } \\
\text { (DIV11) }\end{array}$ \\
\hline MSR $(\mathrm{Hz})$ & $64.5 \pm 2.39$ & $20.5 \pm 1.60$ & $81.3 \pm 5.86$ & $21.4 \pm 0.82$ \\
\hline ISI coefficient of variation & $1.86 \pm 0.029$ & $1.93 \pm 0.046$ & $3.36 \pm 0.15$ & $4.74 \pm 0.10$ \\
\hline Number of active electrodes & $14.4 \pm 0.13$ & $8.68 \pm 0.29$ & $13.3 \pm 0.27$ & $13.4 \pm 0.23$ \\
\hline Number of active wells & 208 & 166 & 201 & 236 \\
\hline MBR $(\mathrm{Hz})$ & $0.44 \pm 0.017$ & $0.16 \pm 0.014$ & $0.69 \pm 0.057$ & $0.77 \pm 0.028$ \\
\hline Number of bursting electrodes & $9.78 \pm 0.28$ & $3.96 \pm 0.28$ & $11.5 \pm 0.43$ & $13.2 \pm 0.23$ \\
\hline Number of bursting wells & 208 & 141 & 172 & 234 \\
\hline Burst duration (s) & $0.98 \pm 0.036$ & $1.15 \pm 0.06$ & $0.97 \pm 0.033$ & $0.32 \pm 0.012$ \\
\hline Number of spikes per burst & $38.9 \pm 1.35$ & $28.7 \pm 1.07$ & $73.3 \pm 3.50$ & $22.7 \pm 0.55$ \\
\hline Mean ISI within burst (s) & $0.03 \pm 0.0007$ & $0.045 \pm 0.002$ & $0.023 \pm 0.001$ & $0.02 \pm 0.001$ \\
\hline IBI (s) & $33.8 \pm 0.97$ & $44.0 \pm 2.22$ & $37.66 \pm 2.47$ & $25.5 \pm 0.94$ \\
\hline IBI coefficient of variation & $0.69 \pm 0.019$ & $0.80 \pm 0.031$ & $0.80 \pm 0.046$ & $0.83 \pm 0.037$ \\
\hline Burst percentage (\%) & $34.8 \pm 0.76$ & $34.7 \pm 1.62$ & $52.3 \pm 1.84$ & $83.3 \pm 0.52$ \\
\hline MNBR (Hz) & $0.1 \pm 0.006$ & $0.08 \pm 0.012$ & $0.074 \pm 0.005$ & $0.073 \pm 0.002$ \\
\hline Network burst duration (s) & $0.92 \pm 0.088$ & $0.88 \pm 0.076$ & $2.12 \pm 0.18$ & $0.75 \pm 0.015$ \\
\hline Number of spikes per network burst & $306 \pm 20.1$ & $166 \pm 22.6$ & $1350 \pm 106$ & $285 \pm 7.83$ \\
\hline Mean ISI within network burst (s) & $0.004 \pm 0.0002$ & $0.007 \pm 0.0004$ & $0.003 \pm 0.0002$ & $0.004 \pm 0.00$ \\
\hline Number of electrodes network burst & $13.2 \pm 0.14$ & $10.1 \pm 0.36$ & $14.4 \pm 0.20$ & $13.6 \pm 0.15$ \\
\hline Number of network bursting wells & 206 & 54 & 154 & 225 \\
\hline Network burst percentage (\%) & $37.1 \pm 1.32$ & $29.8 \pm 2.87$ & $64.7 \pm 2.53$ & $93.0 \pm 0.62$ \\
\hline Network IBI coefficient of variation & $0.73 \pm 0.023$ & $0.56 \pm 0.039$ & $0.83 \pm 0.064$ & $0.78 \pm 0.035$ \\
\hline Network normalized duration IQR & $0.51 \pm 0.043$ & $0.51 \pm 0.091$ & $0.56 \pm 0.13$ & $0.71 \pm 0.030$ \\
\hline Area under normalized cross-correlation & $0.11 \pm 0.003$ & $0.016 \pm 0.002$ & $0.25 \pm 0.012$ & $0.56 \pm 0.011$ \\
\hline FWHH of normalized cross-correlation & $128 \pm 1.60$ & $210 \pm 18.0$ & $734 \pm 21.3$ & $22.8 \pm 0.36$ \\
\hline
\end{tabular}

\subsection{Seizure liability assessment}

Since all models developed spontaneous neuronal activity and (network) bursting behavior, all hiPSC-derived neuronal models were suitable for neurotoxicity testing. To assess their applicability for seizure liability assessment, the different models were exposed to known seizurogenic compounds with different modes of action: strychnine $(0.3-30 \mu \mathrm{M}), 4-\mathrm{AP}(1-100 \mu \mathrm{M})$ and PTX $(0.1-10 \mu \mathrm{M})$. We also exposed rat primary cortical neurons as the current gold standard for MEA seizure liability assessment.

Exposure to the known neurotoxicant strychnine, a glycine receptor antagonist, resulted in an increase of the MSR followed by a decrease at higher test concentrations in the SynFire ${ }^{\circledR}$ iNs co-culture and in the rat primary cortical culture (Fig. 5A, left). However, compared to the rat primary cortical neurons, the in- crease started in the SynFire ${ }^{\circledR}$ iNs co-culture at a 3 times lower concentration. Strychnine significantly decreased the MSR of the iCell ${ }^{\circledR}$ Glutaneurons - iCell ${ }^{\circledR}$ Astrocytes co-culture and the CNS. $4 \mathrm{U}^{\circledR}$ co-culture. MBR increased in the CNS. $4 \mathrm{U}^{\circledR}$ co-culture, SynFire ${ }^{\circledR}$ iNs co-culture, and rat primary cortical culture (Fig. 5B, left). However, this increase was only significant in the latter model. At the highest concentration of strychnine tested, the MBR significantly decreased in the iCell ${ }^{\circledR}$ Glutaneurons - iCell $^{\circledR}$ Astrocytes co-culture and the CNS.4U ${ }^{\circledR}$ co-culture. Strychnine exposure significantly increased the MNBR in the rat primary cortical culture (Fig. 5C, left). However, this was not observed in the hiPSC-derived neuronal models. Burst duration increased in the $\mathrm{CNS} .4 \mathrm{U}^{\circledR}$ co-culture and the $\mathrm{iCell}^{\circledR} \mathrm{Glu}$ taneurons - iCell ${ }^{\circledR}$ Astrocytes co-culture, but only significantly in the latter (Fig. 5D, left). A significant decrease in network 

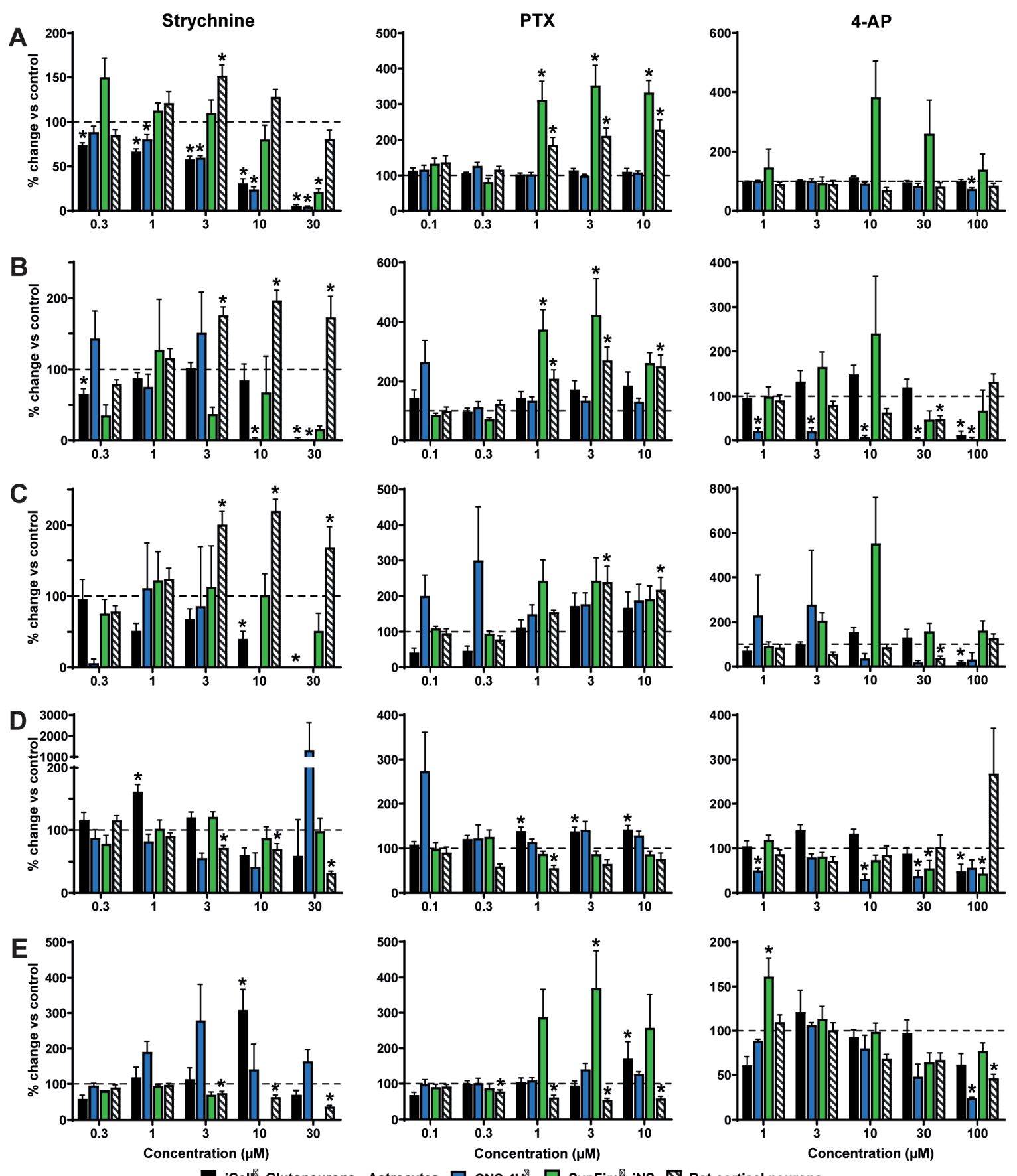

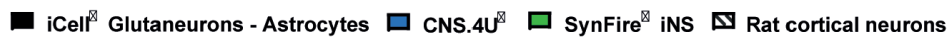

Fig. 5: Toxicological modulation of spontaneous network activity with strychnine (left), PTX (middle) and 4-AP (right) of iCell ${ }^{\circledR}$ Glutaneurons - iCell ${ }^{\circledR}$ Astrocytes co-culture (black), CNS.4U ${ }^{\circledR}$ co-culture (blue), SynFire ${ }^{\circledR}$ iNs co-culture (green) and rat cortical neurons (striped)

Effects are depicted on mean spike rate(MSR; A), mean burst rate (MBR; B), mean network burst rate (MNBR; C), burst duration (D), and network burst duration (E) as average in \% change of control (solvent control set to $100 \%$; dashed line) \pm SEM from $n=3-17$ wells, $\mathrm{N}=2-3$ plates for iCell ${ }^{\circledR}$ Glutaneurons - iCell ${ }^{\circledR}$ Astrocytes co-culture; $n=2-16$ wells, $N=4$ plates for CNS. $4 \mathrm{U}^{\circledR}$ co-culture; $n=3-14$ wells, $\mathrm{N}=4-5$ plates for SynFire ${ }^{\circledR}$ iNs co-culture and $\mathrm{n}=19-29, \mathrm{~N}=5-8$ for rat primary cortical culture. ${ }^{*} p<0.05$. 


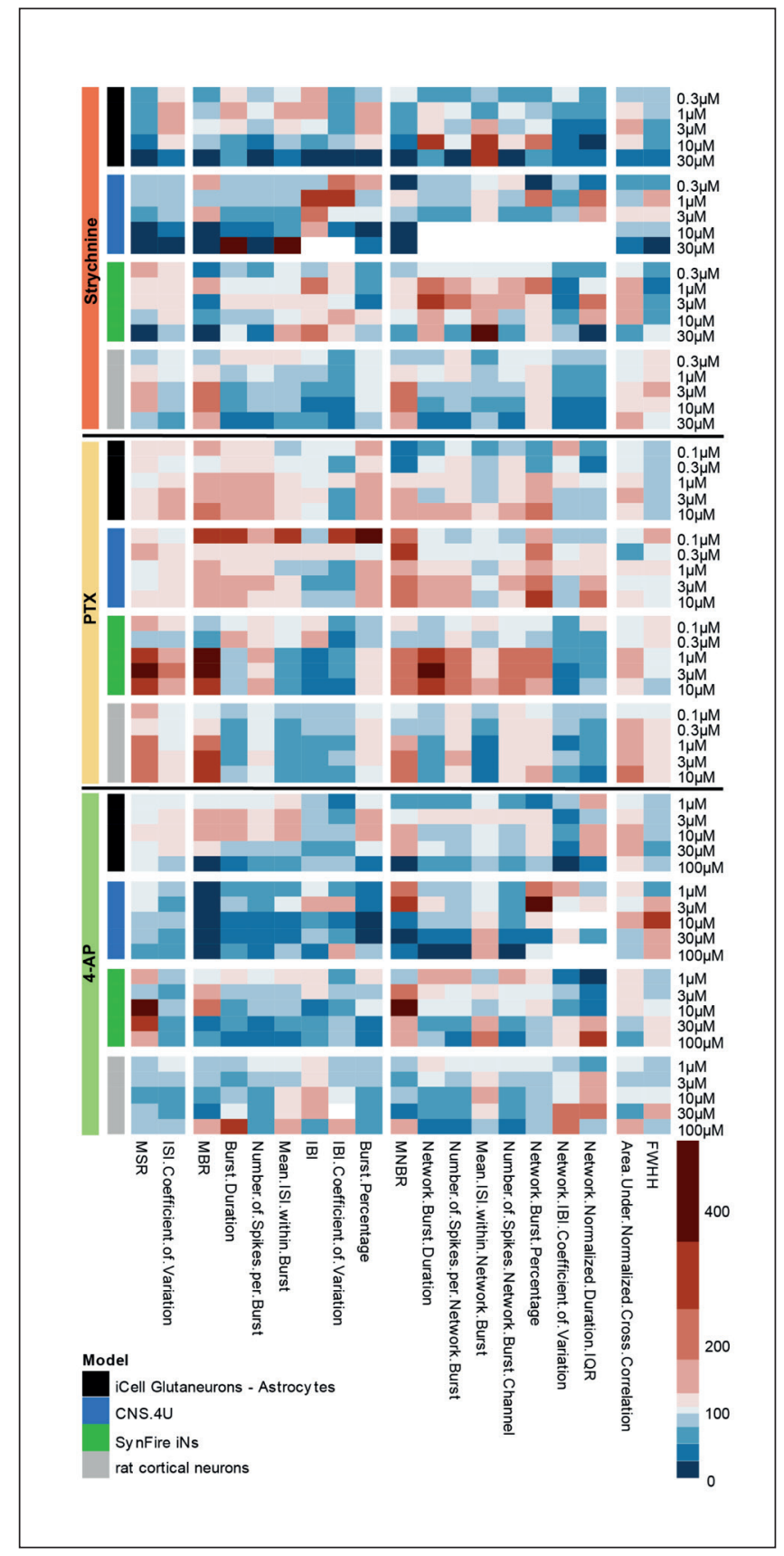

Fig. 6: Heatmap of the effects of strychnine (0.3-30 $\mu \mathrm{M}$; top), PTX (0.1-10 $\mu \mathrm{M}$; middle), and 4-AP (1-100 $\mu \mathrm{M}$; bottom) on selected metric parameters on iCell ${ }^{\circledR}$ Glutaneurons - iCell ${ }^{\circledR}$ Astrocytes co-culture (black), CNS.4U ${ }^{\circledR}$ co-culture (blue), SynFire ${ }^{\circledR}$ iNs co-culture (green), and rat primary cortical neurons (grey)

Color scaling is based on the magnitude of the $\%$ of change relative to the control based on $n=1-18$ wells, $N=2-3$ plates for iCell ${ }^{\circledR}$ Glutaneurons - iCell ${ }^{\circledR}$ Astrocytes co-culture; $n=1-16$ wells, $N=4$ plates for CNS. $4 \mathrm{U}^{\circledR}$ co-culture; $\mathrm{n}=2-15$ wells, $\mathrm{N}=4-5$ plates for SynFire ${ }^{\circledR}$ iNs co-culture and $n=11-30$ wells, $N=5-8$ plates for rat primary cortical culture. For white compartments no average could be calculated. burst duration following strychnine exposure was observed in rat primary cortical neurons (Fig. 5E, left).

PTX, a widely used seizurogenic reference compound and $\mathrm{GABA}_{\mathrm{A}}$-receptor antagonist, showed limited effects on the MSR of iCell ${ }^{\circledR}$ Glutaneurons - iCell ${ }^{\circledR}$ Astrocytes co-culture and CNS.4U ${ }^{\circledR}$ co-culture (Fig. 5A, middle). However, PTX significantly increased the MSR of the SynFire ${ }^{\circledR}$ iNs co-culture and the rat primary cortical culture at higher concentrations. Higher concentrations of PTX significantly increased the MBR of the SynFire ${ }^{\circledR}$ iNs co-culture and the rat primary cortical culture, but had little effect on iCell ${ }^{\circledR}$ Glutaneurons - iCell ${ }^{\circledR}$ Astrocytes co-culture and the CNS.4U ${ }^{\circledR}$ co-culture (Fig. 5B, middle). In all cultures, PTX increased the MNBR (Fig. 5C, middle), but significantly only in the rat primary cortical culture. A significant increase of burst duration was observed for the iCell ${ }^{\circledR}$ Glutaneurons - iCell ${ }^{\circledR}$ Astrocytes co-culture, whereas a decrease occurred in the rat primary cortical culture (Fig. 5D, middle). Network burst duration significantly increased for the iCell ${ }^{\circledR}$ Glutaneurons - iCell ${ }^{\circledR}$ Astrocytes co-culture and the SynFire ${ }^{\circledR}$ iNs co-culture (Fig. 5E, middle). However, network burst duration decreased in the rat primary cortical culture.

4-AP, a known CNS stimulant and potassium channel blocker, only increased the MSR of the SynFire ${ }^{\circledR}$ iNs co-culture (Fig. $5 \mathrm{~A}$, right). MBR increased when the $\mathrm{iCell}^{\circledR}$ Glutaneurons iCell $^{\circledR}$ Astrocytes co-culture and SynFire ${ }^{\circledR}$ iNs co-culture were exposed, although not significantly (Fig. 5B, right). Exposure to 4-AP significantly decreased the MBR of the CNS.4U ${ }^{\circledR}$ co-culture. This is in contrast with the increased MNBR following 4-AP exposure of the CNS.4U ${ }^{\circledR}$ co-culture (Fig. 5C, right). An increase was also observed in the SynFire ${ }^{\circledR}$ iNs co-culture, whereas the iCell ${ }^{\circledR}$ Glutaneurons - iCell ${ }^{\circledR}$ Astrocytes co-culture and rat primary cortical culture were relatively unaffected. Following exposure to 4-AP, burst duration significantly decreased in all hiPSC-derived co-cultures, but this decrease occurred at different concentrations (Fig. 5D, right). Network burst duration increased in the SynFire ${ }^{\circledR}$ iNs co-culture, but decreased in the CNS. $4 \mathrm{U}^{\circledR}$ co-culture and rat primary cortical culture (Fig. 5E, right).

The activity patterns of the different models changed following exposure. More details can be found in the supplementary data 6 (Fig. S2 for strychnine, Fig. S3 for PTX and Fig. S4 for 4-AP).

We created a heatmap of the concentration-response curves of the three test compounds on the four different models to further illustrate the effects on different metric parameters (Fig. 6). By the different color pattern in the heatmap, it becomes clear that strychnine, PTX and 4-AP have different mechanisms of action. For all compounds, but particularly for PTX, the network burst related parameters are most affected when it comes to excitation. Burst related parameters are more sensitive to inhibition especially following 4-AP or strychnine exposure.

Several similarities and differences between the models were observed. Following exposure to strychnine, seizurogenicity was most noticeable in the network burst related parameters in the SynFire ${ }^{\circledR}$ iNs co-culture. Inhibition following strychnine exposure was most pronounced in the CNS. $4 \mathrm{U}^{\circledR}$ co-culture.

When the models were exposed to PTX, all showed a clear 
excitation. The iCell ${ }^{\circledR}$ Glutaneurons - iCell ${ }^{\circledR}$ Astrocytes co-culture and the CNS. $4 \mathrm{U}^{\circledR}$ co-culture reacted in a comparable pattern, whereas the SynFire ${ }^{\circledR}$ iNs co-culture showed more similarities with the rat primary cortical culture.

The only model that is excitable with 4-AP in all three traditional parameters (MSR, MBR and MNBR) is the SynFire ${ }^{\circledR}$ iNs co-culture. The iCell ${ }^{\circledR}$ Glutaneurons - iCell ${ }^{\circledR}$ Astrocytes co-culture shows excitability in burst and network burst parameters at concentrations lower than the rat primary cortical culture. Overall, the SynFire ${ }^{\circledR}$ iNs co-culture was most easily excited, whereas the CNS. $4 \mathrm{U}^{\circledR}$ co-culture was most easily inhibited.

\section{Discussion}

In this study we investigated the applicability of three different human iPSC-derived neuronal models for more predictive in vitro seizure liability assessment without the use of animals or animal tissues.

Our immunocytochemistry data (Fig. 1) demonstrated the mixed nature of the different hiPSC-derived neuronal models. The images show that all models formed highly complex neuronal networks comparable with the ones formed by rat primary cortical cultures (Hondebrink et al., 2016).

All three models developed spontaneous neuronal network activity and (network) bursting behavior over time (Fig. 2-4). However, the level of activity and bursting varied between the different models, despite culturing all models according to the respective manufacturer's protocol. There are several possible explanations for these differences. One likely explanation relates to cell density, as the amount of cells plated in mwMEAs has been shown to greatly influence the spontaneous firing rate, with higher densities resulting in a higher spontaneous activity rate (Jun et al., 2007). In line with this notion, the model with the lowest seeding density $\left(\mathrm{CNS} .4 \mathrm{U}^{\circledR}\right.$ co-culture; Tab. 1) also had the lowest spike and burst activity (Fig. 2). The cell density of the CNS. $4 \mathrm{U}^{\circledR}$ co-culture is also low compared to densities reported in the literature (Odawara et al., 2016; Matsuda et al., 2018; Sasaki et al., 2019). Cell densities of the iCell ${ }^{\circledR}$ Glutaneurons $-\mathrm{iCell}{ }^{\circledR}$ Astrocytes co-culture and, in particular, of the SynFire ${ }^{\circledR}$ iNs co-culture were more in line with densities reported in the literature, and this was paralleled by a higher spontaneous activity level.

The spontaneous firing rate was also enhanced by the presence of astrocytes (Tang et al., 2013; Ishii et al., 2017; Tukker et al., 2018). The higher ratio of astrocytes in the iCell ${ }^{\circledR}$ Glutaneurons - iCell ${ }^{\circledR}$ Astrocytes co-culture and SynFire ${ }^{\circledR}$ iNs co-culture (Tab. 1) may contribute to their higher spike and burst rate (Fig. 2A,B). The SynFire ${ }^{\circledR}$ iNs co-culture was the culture with the highest ratio of astrocytes, which coincided with the highest degree of synchronization (Fig. 4).

Additionally, it has been described previously that human iPSCderived neuronal cultures with a high ratio of glutamatergic neurons exhibit more synchronous bursting events compared to cultures with a low proportion of glutamatergic neurons (Sasaki et al., 2019). During the first culture days, the iCell ${ }^{\circledR}$ Glutaneurons
- iCell ${ }^{\circledR}$ Astrocytes co-culture, which had the highest number of glutamatergic neurons, exhibited the highest MNBR (Fig. 2C). However, at the day of exposure, MNBR was comparable in all cultures. Despite their differences, all the models tested exhibited a pattern of development comparable to that of the rat primary cortical culture (Brown et al., 2016) with MSR, presence of (network) bursts and synchronized activity increasing over time.

Since all models developed spontaneous activity, a neurotoxicity screen was performed in each using the known seizurogenic compounds strychnine, PTX and 4-AP. These compounds are used in in vivo studies to induce seizures (Peña and Tapia, 2000; Mackenzie et al., 2002; Alachkar et al., 2018). Strychnine and 4-AP have also been reported to cause seizures in humans (Pickett and Enns, 1996; van Berlo-van de Laar et al., 2015). Overall, our data indicate the potential of the hiPSC-derived neuronal models for in vitro seizure liability assessment as all three compounds increased activity and synchronicity of the networks.

The potential for seizure liability assessment is further confirmed when comparing our data with two seizure prediction patterns described by Bradley et al. (2018). Seizure prediction pattern 1 is indicative for the response induced by $\mathrm{GABA}_{\mathrm{A}}$-receptor antagonists, such as PTX. This pattern is characterized by an overall increase in activity as well as increased organization and synchronization, reflected in an increased number of spikes per burst, increased interspike interval (ISI) coefficient of variation, increased burst duration, and a decrease in IBI. All four tested models showed an increase in overall activity following exposure to PTX (Fig. 5). Spiking activity increased in a concentration-dependent manner only in the SynFire ${ }^{\circledR}$ iNs co-culture and primary rat cortical neurons, similar to results reported previously for rat primary cortical cultures (Mack et al., 2014; Kreir et al., 2018) and hiPSC-derived neuronal co-cultures (Kreir et al., 2019). An increase in activity was observed for all models in (network) bursting, comparable to results from Kreir et al. (2019). Also, organization and synchronization were enhanced according to prediction pattern 1 (Fig. 6).

Seizure prediction pattern 2 is characterized by a decrease in overall activity and a deterioration of network organization, reflected in an increased IBI, a decreased number of spikes per burst, and a decreased ISI coefficient of variation and burst duration. This pattern is indicative of exposure to the glycine receptor antagonist strychnine (Bradley et al., 2018). The iCell ${ }^{\circledR}$ Glutaneurons - $\mathrm{iCell}^{\circledR}$ Astrocytes co-culture and CNS.4U ${ }^{\circledR}$ co-culture exhibited decreased activity (Fig. 5) and network disintegration (Fig. 6) following the described prediction pattern. Changes in spike and burst activity at the lowest test concentration in the $\mathrm{iCell}^{\circledR}$ Glutaneurons - iCell ${ }^{\circledR}$ Astrocytes co-culture are statistically significant, indicating a high sensitivity of this model for the tested glycine receptor agonist. However, this does not necessarily indicate biological relevance. In contrast to earlier reported findings (Bradley et al., 2018; Kreir et al., 2018), activity of the rat primary cortical culture increased following strychnine exposure. However, this could be due to differences in maturation stage between E18-19 and PND1 cortices. During embryonic development, glycine receptors have an excitatory function, whereas they become inhibitory later on 
(Dutertre et al., 2012).

Exposure to the potassium channel blocker 4-AP is reported to follow seizure prediction pattern 1 (Bradley et al., 2018), however our data do not support that notion (Fig. 5, 6). An increase in overall activity is only observed in the SynFire ${ }^{\circledR}$ iNs co-culture and an increase in bursting activity in the iCell ${ }^{\circledR}$ Glutaneurons - iCell ${ }^{\circledR}$ Astrocytes co-culture (Fig. 5), but the increased organization and synchronization to match pattern 1 is not present (Fig. 6). Kreir et al. (2019) reported an increase in spike and network burst frequency following exposure of hiPSC-derived neuronal cultures to strychnine. This matches our SynFire ${ }^{\circledR}$ iNS co-culture data. The other cultures showed a decreased activity pattern, but with a network deterioration that did not match prediction pattern 2. Rat hippocampal neurons cultured on MEA plates exhibit increased activity (Fan et al., 2019). It could thus be that a more hippocampal-like phenotype is required to detect the seizurogenic activity of 4-AP.

When comparing the differences in sensitivity of the models, the aforementioned points with regard to ratio of excitatory to inhibitory neurons, number of astrocytes present, and seeding density must be kept in mind, since these can all affect chemical sensitivity. Although cells were exposed when they exhibited network maturation and according to continuously improving manufacturer's protocols, network complexity (e.g., the number of contacts that the different cell types establish) can still be different among the different models. This may influence how the models react to chemical insults. Also, receptor expression may differ between the different hiPSC-derived neuronal models. Spontaneous activity and drug responses are dictated by the complex interplay of a large number of different receptor types, ion channels and transporters, all consisting of different subtypes. To better understand the differences in chemical sensitivity between the models, full protein profiles of the expression levels of the different receptors, channels and transporters present must be made. However, not only the numbers of receptors, channels and transporters are crucial, also the interplay, location and distribution of the receptors, channels and transporters define chemical sensitivity.

We showed that all tested models were able to form spontaneously active networks with (network) bursting behavior. Our data also indicated that these models can be used for animal-free in vitro seizure liability assessment. More importantly, the iPSCderived models were capable of modeling seizure-like activity at the same level or even better than the rat primary cortical neuronal culture. However, in order to detect seizure-like activity in vitro with a MEA system, multiple parameters must be assessed. When only spike behavior is investigated, seizure-like activity may be missed.

Also, when comparing data from hiPSC-derived neuronal models with rat primary cortical cultures, it must be kept in mind that there are differences between rodents and humans. Hence, rodent experiments are not always predictive for human risk (Olson et al., 2000). It is thus logical that results can differ between in vitro assays that are performed using human cells and assays using rodent cells (Hondebrink et al., 2017). This in turn results in different hits and sensitivities (Malik et al., 2014).
However, there are also cases where the rodent and human system show comparable results (Kasteel and Westerink, 2017). The discrepancies between the rat primary rodent cortical culture and our hiPSC-derived neuronal models should not be the main concern. Rather, attention must be focused on further characterization and optimization of hiPSC-derived neuronal models to make them more closely resemble the human brain, specifically those parts that are involved in epileptic activity such as the amygdala, hippocampus and neocortex (Rogawski and Löscher, 2004).

Also, it must be kept in mind that this study was done with a limited number of test compounds. To fully understand the applicability of hiPSC-derived neuronal models for in vitro seizure liability assessment, a broader set of compounds should be tested, including, besides seizurogenics, anti-seizurogenic and negative compounds.

Nevertheless, the current data show that hiPSC-derived neuronal models may already be used as a first screen for epileptic activity before performing follow-up studies. With that we are one step closer to animal-free in vitro seizure liability assessment. To fully move away from animal testing for in vitro seizure liability assessment, hiPSC-models must be developed in which all targets involved in the onset and duration of seizures are present and validated using a large library of diverse reference compounds. With respect to the comparison of hiPSC-derived neuronal cultures with rodent primary neuronal cultures; such a comparison cannot be performed one on one. Actually, our data show some striking differences between the different models, highlighting the need to move to human model systems, thereby eliminating the need for interspecies extrapolation.

\section{References}

Alachkar, A., Łażewska, D., Latacz, G. et al. (2018). Studies on anticonvulsant effects of novel histamine H3R antagonists in electrically and chemically induced seizures in rats. Int $\mathrm{J} \mathrm{Mol}$ Sci 19, 3386. doi:10.3390/ijms19113386

Anson, B. D., Kolaja, K. L. and Kamp, T. J. (2011). Opportunities for use of human iPS cells in predictive toxicology. Clin Pharmacol Ther 89, 754-758. doi:10.1038/clpt.2011.9

Authier, S., Arezzo, J., Delatte, M. S. et al. (2016). Safety pharmacology investigations on the nervous system: An industry survey. J Pharmacol Toxicol Methods 81, 37-46. doi:10.1016/J. VASCN.2016.06.001

Bradley, J. A., Luithardt, H. H., Metea, M. R. et al. (2018). In vitro screening for seizure liability using microelectrode array technology. Toxicol Sci 163, 240-253. doi:10.1093/toxsci/ kfy029

Brown, J. P., Hall, D., Frank, C. L. et al. (2016). Evaluation of a microelectrode array-based assay for neural network ontogeny using training set chemicals. Toxicol Sci 154, 126-139. doi:10.1093/toxsci/kfw147

Buskila, Y., Breen, P. P., Tapson, J. et al. (2015). Extending the viability of acute brain slices. Sci Rep 4, 5309. doi:10.1038/ srep05309

Cotterill, E., Charlesworth, P., Thomas, C. W. et al. (2016). A 
comparison of computational methods for detecting bursts in neuronal spike trains and their application to human stem cell-derived neuronal networks. J Neurophysiol 116, 306-321. doi:10.1152/jn.00093.2016

Dingemans, M. M. L., Schütte, M. G., Wiersma, D. M. M. et al. (2016). Chronic 14-day exposure to insecticides or methylmercury modulates neuronal activity in primary rat cortical cultures. Neurotoxicology 57, 194-202. doi:10.1016/j.neuro. 2016.10.002

Dutertre, S., Becker, C.-M. and Betz, H. (2012). Inhibitory glycine receptors: An update. J Biol Chem 287, 40216-40223. doi:10.1074/jbc.R112.408229

Easter, A., Bell, M. E., Damewood, J. R. et al. (2009). Approaches to seizure risk assessment in preclinical drug discovery. Drug Discov Today 14, 876-884. doi:10.1016/j. drudis.2009.06.003

Fan, J., Thalody, G., Kwagh, J. et al. (2019). Assessing seizure liability using multi-electrode arrays (MEA). Toxicol In Vitro 55, 93-100. doi:10.1016/j.tiv.2018.12.001

Görtz, P., Fleischer, W., Rosenbaum, C. et al. (2004). Neuronal network properties of human teratocarcinoma cell line-derived neurons. Brain Res 1018, 18-25. doi:10.1016/j.brainres. 2004.05.076

Grainger, A. I., King, M. C., Nagel, D. A. et al. (2018). In vitro models for seizure-liability testing using induced pluripotent stem cells. Front Neurosci 12, 590. doi:10.3389/ fnins.2018.00590

Hay, M., Thomas, D. W., Craighead, J. L. et al. (2014). Clinical development success rates for investigational drugs. Nat Biotechnol 32, 40-51. doi:10.1038/nbt.2786

Hogberg, H. T., Sobanski, T., Novellino, A. et al. (2011). Application of micro-electrode arrays (MEAs) as an emerging technology for developmental neurotoxicity: Evaluation of domoic acid-induced effects in primary cultures of rat cortical neurons. Neurotoxicology 32, 158-168. doi:10.1016/j.neuro. 2010.10.007

Hondebrink, L., Verboven, A. H. A., Drega, W. S. et al. (2016). Neurotoxicity screening of (illicit) drugs using novel methods for analysis of microelectrode array (MEA) recordings. Neurotoxicology 55, 1-9. doi:10.1016/j.neuro.2016.04.020

Hondebrink, L., Kasteel, E. E. J., Tukker, A. M. et al. (2017). Neuropharmacological characterization of the new psychoactive substance methoxetamine. Neuropharmacology 123, 1-9. doi:10.1016/j.neuropharm.2017.04.035

Ishii, M. N., Yamamoto, K., Shoji, M. et al. (2017). Human induced pluripotent stem cell (hiPSC)-derived neurons respond to convulsant drugs when co-cultured with hiPSC-derived astrocytes. Toxicology 389, 130-138. doi:10.1016/j.tox. 2017.06.010

Jiruska, P., de Curtis, M., Jefferys, J. G. R. et al. (2013). Synchronization and desynchronization in epilepsy: Controversies and hypotheses. J Physiol 591, 787-797. doi:10.1113/jphysiol. 2012.239590

Johnstone, A. F. M., Gross, G. W., Weiss, D. G. et al. (2010). Microelectrode arrays: A physiologically based neurotoxicity testing platform for the $21^{\text {st }}$ century. Neurotoxicology 31,331 -
350. doi:10.1016/j.neuro.2010.04.001

Jun, S. B., Hynd, M. R., Dowell-Mesfin, N. et al. (2007). Low-density neuronal networks cultured using patterned poly-l-lysine on microelectrode arrays. J Neurosci Methods 160, 317-326. doi:10.1016/j.jneumeth.2006.09.009

Kasteel, E. E. J. and Westerink, R. H. S. (2017). Comparison of the acute inhibitory effects of tetrodotoxin (TTX) in rat and human neuronal networks for risk assessment purposes. Toxicol Lett 270, 12-16. doi:10.1016/j.toxlet.2017.02.014

Kreir, M., Van Deuren, B., Versweyveld, S. et al. (2018). Do in vitro assays in rat primary neurons predict drug-induced seizure liability in humans? Toxicol Appl Pharmacol 346, 45-57. doi:10.1016/J.TAAP.2018.03.028

Kreir, M., De Bondt, A., Van den Wyngaert, I. et al. (2019). Role of Kv7.2/Kv7.3 and M1 muscarinic receptors in the regulation of neuronal excitability in hiPSC-derived neurons. Eur J Pharmacol, 172474. doi:10.1016/j.ejphar.2019.172474

Kuijlaars, J., Oyelami, T., Diels, A. et al. (2016). Sustained synchronized neuronal network activity in a human astrocyte co-culture system. Sci Rep 6, 36529. doi:10.1038/srep36529

Legéndy, C. R. and Salcman, M. (1985). Bursts and recurrences of bursts in the spike trains of spontaneously active striate cortex neurons. $J$ Neurophysiol 53, 926-939. doi:10.1152/ jn.1985.53.4.926

Little, D., Ketteler, R., Gissen, P. et al. (2019). Using stem cell-derived neurons in drug screening for neurological diseases. Neurobiol Aging 78, 130-141. doi:10.1016/j.neurobiolaging. 2019.02.008

Mack, C. M., Lin, B. J., Turner, J. D. et al. (2014). Burst and principal components analyses of MEA data for 16 chemicals describe at least three effects classes. Neurotoxicology 40, 75-85. doi:10.1016/j.neuro.2013.11.008

Mackenzie, L., Medvedev, A., Hiscock, J. J. et al. (2002). Picrotoxin-induced generalised convulsive seizure in rat: Changes in regional distribution and frequency of the power of electroencephalogram rhythms. Clin Neurophysiol 113, 586-596. doi:10.1016/S1388-2457(02)00040-8

Malik, N., Efthymiou, A. G., Mather, K. et al. (2014). Compounds with species and cell type specific toxicity identified in a 2000 compound drug screen of neural stem cells and rat mixed cortical neurons. Neurotoxicology 45, 192-200. doi: 10.1016/j.neuro.2014.10.007

Markram, H., Toledo-Rodriguez, M., Wang, Y. et al. (2004). Interneurons of the neocortical inhibitory system. Nat Rev Neurosci 5, 793-807. doi:10.1038/nrn1519

Matsuda, N., Odawara, A., Katoh, H. et al. (2018). Detection of synchronized burst firing in cultured human induced pluripotent stem cell-derived neurons using a 4-step method. Biochem Biophys Res Commun 497, 612-618. doi:10.1016/j.bbrc. 2018.02.117

McConnell, E. R., McClain, M. A., Ross, J. et al. (2012). Evaluation of multi-well microelectrode arrays for neurotoxicity screening using a chemical training set. Neurotoxicology 33, 1048-1057. doi:10.1016/j.neuro.2012.05.001

Nicolas, J., Hendriksen, P. J. M., van Kleef, R. G. D. M. et al. (2014). Detection of marine neurotoxins in food safety test- 
ing using a multielectrode array. Mol Nutr Food Res 58, 23692378. doi:10.1002/mnfr. 201400479

Odawara, A., Katoh, H., Matsuda, N. et al. (2016). Physiological maturation and drug responses of human induced pluripotent stem cell-derived cortical neuronal networks in long-term culture. Sci Rep 6, 26181. doi:10.1038/srep26181

Odawara, A., Matsuda, N., Ishibashi, Y. et al. (2018). Toxicological evaluation of convulsant and anticonvulsant drugs in human induced pluripotent stem cell-derived cortical neuronal networks using an MEA system. Sci Rep 8, 10416. doi: 10.1038/s41598-018-28835-7

Olson, H., Betton, G., Robinson, D. et al. (2000). Concordance of the toxicity of pharmaceuticals in humans and in animals. Regul Toxicol Pharmacol 32, 56-67. doi:10.1006/rtph.2000.1399

Onakpoya, I. J., Heneghan, C. J. and Aronson, J. K. (2016). Post-marketing withdrawal of 462 medicinal products because of adverse drug reactions: A systematic review of the world literature. BMC Med 14, 10. doi:10.1186/s12916-016-0553-2

Paavilainen, T., Pelkonen, A., Mäkinen, M. E.-L. et al. (2018). Effect of prolonged differentiation on functional maturation of human pluripotent stem cell-derived neuronal cultures. Stem Cell Res 27, 151-161. doi:10.1016/j.scr.2018.01.018

Peña, F. and Tapia, R. (2000). Seizures and neurodegeneration induced by 4-aminopyridine in rat hippocampus in vivo: Role of glutamate- and GABA-mediated neurotransmission and of ion channels. Neuroscience 101, 547-561. doi:10.1016/S03064522(00)00400-0

Pickett, T. A. and Enns, R. (1996). Atypical presentation of 4-aminopyridine overdose. Ann Emerg Med 27, 382-385. doi:10.1016/S0196-0644(96)70277-9

Rogawski, M. A. and Löscher, W. (2004). The neurobiology of antiepileptic drugs. Nat Rev Neurosci 5, 553-564. doi:10.1038/ nrn 1430

Sasaki, T., Suzuki, I., Yokoi, R. et al. (2019). Synchronous spike patterns in differently mixed cultures of human iPSC-derived glutamatergic and GABAergic neurons. Biochem Biophys Res Commun 513, 300-305. doi:10.1016/j.bbrc.2019.03.161

Tang, X., Zhou, L., Wagner, A. M. et al. (2013). Astroglial cells regulate the developmental timeline of human neurons differentiated from induced pluripotent stem cells. Stem Cell Res 11, 743-757. doi:10.1016/j.scr.2013.05.002
Tukker, A. M., De Groot, M. W. G. D. M., Wijnolts, F. M. J. et al. (2016). Is the time right for in vitro neurotoxicity testing using human iPSC-derived neurons? ALTEX 33, 261-271. doi:10.14573/altex.1510091

Tukker, A. M., Wijnolts, F. M. J., de Groot, A. et al. (2018). Human iPSC-derived neuronal models for in vitro neurotoxicity assessment. Neurotoxicology 67, 215-225. doi:10.1016/j.neuro. 2018.06.007

Tukker, A. M., Wijnolts, F. M. J., de Groot, A. et al. (2019). In vitro techniques for assessing neurotoxicity using human iPSCderived neuronal models. In M. Aschner and L. Costa (eds.), Cell Culture Techniques. Neuromethods (17-35). Vol. 145. New York, NY: Humana. doi:10.1007/978-1-4939-9228-7_2

Valdivia, P., Martin, M., LeFew, W. R. et al. (2014). Multi-well microelectrode array recordings detect neuroactivity of ToxCast compounds. Neurotoxicology 44, 204-217. doi:10.1016/j. neuro.2014.06.012

van Berlo-van de Laar, I. R. F., Arbouw, M. E. L. and Bles, C. M. A. (2015). Strychnine poisoning: Uncommon, but does still happen. Ned Tijdschr Geneeskd 159, A8877. http://www.ncbi. nlm.nih.gov/pubmed/26173662 (accessed 17.09.2019).

Westerink, R. H. S. (2013). Do we really want to REACH out to in vitro? Neurotoxicology 39, 169-172. doi:10.1016/j.neuro.2013. 10.001

\section{Conflict of interest}

The authors declare that they have no conflict of interest.

\section{Acknowledgements}

We gratefully acknowledge members of the Neurotoxicology Research Group for helpful discussions. We thank Richard Wubbolts and Esther van 't Veld (Center for Cell Imaging, Utrecht University) for help with the microscopy work and Barbara Vreede (University Library, Utrecht University) for assisting with $\mathrm{R}$ programming of the heatmap. This work was funded by a grant from the National Centre for the Replacement, Refinement and Reduction of Animals in Research (NC3Rs; project number 50308-372160) and by the Faculty of Veterinary Medicine (Utrecht University, The Netherlands). 